\title{
La conquête du « vide » ou la nécessité d'être nomade chez les
} Touaregs.

Hélène Claudot-Hawad

\section{Citer ce document / Cite this document :}

Claudot-Hawad Hélène. La conquête du « vide » ou la nécessité d'être nomade chez les Touaregs.. In: Revue de l'Occident musulman et de la Méditerranée, n41-42, 1986. Désert et montagne au Maghreb. pp. 397-412;

doi : 10.3406/remmm.1986.2469

http://www.persee.fr/doc/remmm_0035-1474_1986_num_41_1_2469

Document généré le 07/06/2016 
Hélène Claudot-Hawad

\title{
LA CONQUÊTE DU "VIDE » OU LA NÉCESSITÉ D'ÊTRE NOMADE CHEZ LES TOUAREGS
}

\author{
Du puits à la tente \\ de la tente au vide \\ le sentier s'enroule \\ anneau serpent \\ gémissement aigu \\ de la flûte \\ qui rassemble les fibres \\ du visible et de l'invisible \\ Pour incendier le mystère \\ du couchant \\ le feu naquit \\ de la source du levant

Hawad

Ténéré en touareg signifie le "désert", c'est-à-dire le lieu désolé et stérile qui n'autorise la survie ni des hommes ni de leurs troupeaux. Il représente le territoire dangereux de l'étrange, de l'inconnu, de l'insaisissable, du sauvage, du surnaturel, où l'homme ne peut imprimer ses traces ni marquer son chemin. C'est le domaine privilégié des génies (Kel ténéré) qui menacent les êtres de l'espace domestique. C'est l'abri d'élection du monde de l' "extérieur ", appelé essuf, qui s'oppose à l'« intérieur " auquel s'identifient les humains, mais qui en est également l'indispensable contrepartie. L'univers, en effet, est construit sur ces deux axes, antagonistes et indissociables, qui se croisent comme les arceaux d'une tente. 
Le ténéré ne représente que des franges bordant et séparant les territoires habités par les nomades. Il trace une frontière instable que des efforts constants doivent toujours repousser. Car le vide, l'essuf, menace toute chose. Il peut envahir par exemple un lieu temporairement délaissé, déserté, comme le puits à la tombée de la nuit, qui ne sera plus fréquenté, alors, que par les voyageurs étrangers, les animaux sauvages ou les êtres errants. Il peut aussi s'emparer d'une personne qui s'est laissée égarer à la croisée des chemins...

Tentant d'échapper aux dangers de cet «extérieur " qui les guettent à chaque carrefour, les voyageurs cosmiques que représentent les hommes tout comme les animaux, les plantes, les choses, les éléments, les moindres petites particules qui participent au monde de l'intelligible, accomplissent un périple jalonné d'étapes fixes et obligatoires. C'est précisément de cet espace aménagé, de ce faux désert où s'inscrit et s'ordonne le cycle de la vie comme celui des transhumances, parcours à la fois matériel et spirituel, qu'il sera question ici.

\section{L'ABRI}

Selon la pensée touarègue, aucun être, aucun objet ne saurait exister sans la protection d'un abri. Ainsi, les grains de sable ont besoin des failles du sol ou du roc pour se stabiliser, le fennec se réfugie dans son terrier, l'aigle dans son nid, l'homme dans sa tente pour échapper aux tempêtes de l'kextérieur ». Cependant, l'abri luimême deviendrait inutile sans la proximité de l'eau. Le "chemin qui mène au puits" (tézezregt) est le parcours obligatoire qui relie les êtres à la vie. Végétaux, animaux sauvages ou domestiques, humains, tous se retrouvent sur le sentier de l'eau. A l'image du parcours quotidien de la tente au puits et du puits à la tente qui chaque nuit ramène le nomade à son point de départ, tout être suit un cycle dont l'achèvement marque le début d'un autre cycle, parcours indéfiniment répété. Toutes les actions, tous les mouvements qui animent les êtres, les choses, les éléments, aboutissent à ces deux points d'ancrage que sont l'eau et l'abri.

Pour un nomade, le cœur de l'espace habité est d'abord sa tente, c'est-à-dire la tente où il est né, celle de sa mère. En effet, dans la majorité des groupes touaregs, la règle de filiation est matrilinéaire et les enfants sont rattachés à la lignée de leur mère. Par ailleurs, la tente est un bien féminin, constitué au moment du mariage. Chaque nouvelle tente doit comprendre au moins quelques éléments de la tente maternelle, prolongation symbolique de l'abri originel qui permit aux ancêtres de la famille d'"être".

Le terme éhen, "tente", s'utilise également pour désigner métaphoriquement l' "épouse ", détentrice de la tente de peau ou de nattes qui abrite le couple. Abri temporaire pour l'homme cette fois puisqu'en cas de divorce ou de veuvage, il perdra simultanément épouse et logement. Au contraire, la femme ne "sort " jamais de l'abri; elle se confond quelquefois avec lui. Et si elle se marie à l'extérieur de son clan (appelé tawshit et conçu pour la plupart des cas comme un groupe de descendance matrilinéaire), elle va créer une véritable enclave territoriale dans le campement de son mari en y installant sa tente qui certes lui appartient en propre mais représente également sa lignée toute entière. 
L'un des principes cher à la société touarègue est celui de l'autonomie économique de la femme qui en général (à l'exception des suzerains Iwellemmeden) arrive chez ses alliés munie non seulement de sa tente et de tous les ustensiles domestiques nécessaires, mais aussi de troupeaux, de biens, de serviteurs en nombre suffisant pour la faire vivre complètement indépendamment de son époux si elle le désire. L'indépendance de cette femme par rapport à ses alliés garantit en même temps l'indépendance de la lignée qu'elle représente.

Enfin, éhen désigne la famille utérine descendant de cette ancêtre "mère * qui fonda l'abri et assura ainsi le devenir de sa lignée. Le terme s'applique plus particulièrement à l'ensemble des femmes qui constituent ce noyau matrilinéaire de la parenté, axe suivant lequel sont transmis des droits ou des biens, inaliénables, qui, de la même façon que l'abri, vont assurer la survie de la communauté. Le terme équivaut également dans ce sens à ébawél ou ébategh, qui peuvent dénommer l'abri que constitue la famille utérine, s'appliquant aussi à l'ancêtre féminine matrilinéaire et enfin à la tente qui, transmise de mère en fille, marque la continuité de la famille (voir $\mathrm{H}$. Claudot et $M$. Hawad, 1984).

Parmi les attributs indispensables qui permettent l'établissement d'une lignée et garantissent son existence, figurent les droits territoriaux, qui sont d'ailleurs une simple extension de l'abri (notons que la notion d'ébawél sert aussi à évoquer le territoire, c'est-à-dire le pays natal ou pays de la mère). Aux différents embranchements du matrilignage, figuré comme un emboîtement de petites tentes dont l'ensemble forme la grande tente ou tente de la mère (éhen $n \mathrm{ma}$ ), correspondent des espaces de nomadisation priviliégiés, mais inaliénables.

En fait, il en est des droits d'accès au territoire comme des droits d'usage des biens d'ébawél appelés le "lait " d'ébawél (akh n ébawél, voir H. Claudot et $\mathrm{M}$. Hawad, 1984a, 1984b) et destinés à * nourrir * la lignée, c'est-à-dire à en assurer la subsistance et la perpétuation. Lorsqu'une fille de la parenté se marie, c'est-à-dire chaque fois qu'une nouvelle tente s'établit, une part des biens de la "grande tente (ou grand ébawél) lui est attribuée pour qu'elle puisse survivre; mais cette cession est temporaire, car le jour où le mariage est dénoué, la femme, sa tente et les biens qui lui permettent de jouer le rôle d'abri, reviendront à leur point de départ, devenant abri dans l'abri.

Ainsi, la sociétê peut être vue comme une superposition infinie de tentes, tandis que l'aîné(e) du cercle de parenté est censé jouer le rôle du pilier central (tamenkayt) qui soutient le velum, entouré et assisté par les piquets latéraux.

A l'enclavement inclusif de ces abris-tentes, correspond l'emboîtement étroit des biens et des droits qui les fondent. Car chaque tente constitue en même temps le piquet d'une tente plus vaste, le bien de chaque petit ébawél est aussi une part du «lait " qui nourrit le grand ébawél, le territoire complet qu'exploite un campement représente un simple élément du grand territoire nécessaire à la confédération.

\section{DES REFUGES DISPARATES}

Chaque individu, par son appartenance à un groupe de descendance qui se représente comme un ensemble de parents utérins, devrait en principe avoir accès aux 
droits et aux biens transmis, depuis l'ancêtre fondatrice, en voie matrilinéaire. Mais, en fait, les pratiques de mariage endogame à l'intérieur du matrilignage ou de la "tente" aboutissent (voir $\mathrm{H}$. Claudot, 1980, 1983, 1986) à une réduction du cercle des ayants droit et à une hiérarchisation au sein du groupe de descendance. Par ailleurs, en dépit de la règle matrilinéaire énoncée, le recrutement des membres du groupe de filiation est bilatéral (la tente la plus forte ou la plus influente capte les enfants issus d'un mariage mixte).

Il en résulte une inégalité de fait entre les différentes tentes qui composent la société. La division sociale entre les gens du "pouvoir" et les autres sera interprétée sur le mode parental par une opposition entre enfants de sœur et enfants de frères ou entre aînés et cadets, les diverses parties restant liées à l'ensemble par le réseau généalogique. Ou encore, une vision plus politique de l'organisation sociale superposera au ciment parental des unités, un axe délibérément hiérarchique qui tranche l'ensemble social en strates irréductibles.

Ainsi, le chef de la confédération, amenukal, peut être défini comme appartenant au groupe suzerain dominant et chef d'une unité politique stratifiée, mais il peut être considéré également comme l'aîné de la tente aînée, elle-même à la tête des autres tentes, et se trouvant, pour cette raison, responsable des biens de la communauté toute entière, qui doivent rester indivis. Beaucoup de Touaregs pensent que le terme amenukal signifie "celui qui tient ou qui possède le pays". La véracité de cette étymologie, contestée par certains linguistes, importe moins ici que la représentation qu'elle véhicule et qui conforte l'idée de l'indivision finale des biens qui assurent la survie du groupe et dont la responsabilité incombe à l'aîné de ses membres. Ainsi, de la même façon que l'abri-mère concède une partie de ses biens au petit abri nouvellement né pour qu'il assure sa survie en même temps que la prolongation de la lignée dont il est issu, de même des droits privilégiés sur une portion de l'espace de la grande tente sont attribués aux petites tentes.

En fait, chaque unité sociale, du foyer conjugal à la confédération ou à la société toute entière, contient le ferment de cette inégalité, et reproduit sur le même mode le schéma de distribution des biens et des droits. Si certaines "tentes", héritières directes de la "tente" d'origine, ont accès à la chefferie et à la gestion des moyens de production de la communauté, d'autres, déclassées, en sont écartées, tout en conservant, au niveau de leur tente restreinte, une certaine autonomie.

Le rapport de force entre les tentes est traduit dans les généalogies par un lien de parenté dissymétrique établi entre les ancêtres fondateurs (aîné-cadet; enfant de sœur-enfant de frère), qui légitime ainsi l'opposition entre la tente de la chefferie et les autres tentes, ou encore entre suzerains et tributaires... (voir $\mathrm{H}$. Claudot 1982, 56-65).

Dans les groupes matrilinéaires (que nous prendrons seuls en compte dans cet article), le regroupement résidentiel obéit à plusieurs principes : la première étape pour un individu, si ses parents ne sont ni divorcés ni décédés, est généralement patrilocale. A la mort de son père ou au moment du divorce de ses parents, un enfant, s'il est petit, suit sa mère et retourne dans le campement d'origine de cette dernière, chez son oncle maternel. Enfin, lorsqu'une femme se marie, elle part la plupart du temps s'installer chez son époux où elle demeure jusqu'à ce que l'alliance soit dissoute.

Dans certains groupes, l'étape viri-locale n'existe pas ou bien se trouve annulée 
par la nature endogame du mariage (à l'intérieur de la matriligne). De même, la patri- et la matri-localité, lorsque le père et la mère sont de proches parents matrilinéaires, sont confondues. Mais le principe essentiel qui anime les mouvements résidentiels est partout matrilinéaire et renvoie à nouveau à ce parcours cyclique que les individus accomplissent pour revenir inévitablement à leur point de départ, à leur tente d'origine. Si les femmes affrontent l'« extérieur " en transportant leur abri pour en principe revenir au grand abri, les hommes sont plus démunis : lorsque, adolescents, ils sortent de la tente de leur mère, ils doivent traverser l'essuf sans protection. Cette épreuve fait partie de l'initiation des jeunes gens qui doivent passer une nuit dans le désert, hors de l'espace domestiqué. Seuls résisteront aux dangers de l'" extêrieur" ceux qui ont acquis suffisamment de connaissances sur l'ordre du monde. Ils sont alors aptes à franchir une autre étape du cycle social en «nouant une tente ", c'est-à-dire en se mariant et trouvant, après une nouvelle traversée de l'essuf, un refuge qu'ils s'efforceront d'entraîner et d'ancrer sur leur territoire.

Ainsi, les droits d'usage sur le territoire sont théoriquement déterminés par l'appartenance à un groupe de filiation plus ou moins fictif comprenant plusieurs divisions : chaque sous-ensemble correspond à un cercle de parenté, du plus étroit et concret au plus large et hypothétique, qui va de la "tente-foyer" à la "tentefraction ", s'étendant à la "tente-tribu ", à la "tente-confédération ", à la "tentesociété " jusqu'à la "tente-univers "... Les tentes "aînées" forment le pilier de la société, dirigeant l'ensemble et gérant ses resssources afin de maintenir l'édifice social, toujours menacé par le "désert" environnant. Au nom de leur ancienneté, réelle ou décrétée, elles tiennent en main le capital indivis de la collectivité, mais assurent en même temps une certaine autonomie aux jeunes tentes en leur octroyant un droit privilégié bien que considéré comme temporaire sur une partie de ces biens, en théorie non cessibles et non destructibles.

\section{LE TERRITOIRE}

Un jour, une femme de la tribu des Uraghen de l'Ajjer, accompagnée de ses deux sœurs, arriva dans l'Ayr. Les trois femmes furent accueillies par les Kel Tamat, nobles de ce pays. L'un d'entre eux offrit le mariage à l'aînée des filles qui accepta à condition que sa taggalt (compensation matrimoniale) soit convertie en sol. Un guerrier valeureux devait partir dès la pointe de l'aube sur un cheval des Bagzan (coursier réputé) mené au galop; au coucher du soleil, il arrêterait alors sa monture et lancerait son javelot au loin, droit devant lui. Fichée en terre, la pointe de la lance du cavalier marquerait la limite du territoire.

Les sœurs et les filles de cette femme, qui sont à l'origine de la tribu des Ikazkazen, exigèrent les mêmes dots et les Kel Tamat finirent par perdre leur pays et s'exiler au Damergu.

Ce mythe, dont les variantes régionales foisonnent, associe bien tous les ingrédients utiles à la fondation d'une tente : ainsi, une ou plusieurs femmes apparentées arrivent qui donneront naissance à un groupe nouveau, et acquièrent un territoire ou souvent encore du bétail qu'elles transmettront à leur descendance. Ce 


\section{2 / H. Claudot-Hawad}

capital primordial est obtenu de gré ou de force, par alliance, par achat, par don ou par conquête (voir par exemple la version du chef des Imenan, Guma, distribuant les terres aux dames des Ajjer, in Duveyrier, 1864 : 324, ou encore, Ti-nHinan, l'ancêtre des suzerains de l'Ahaggar, donnant à des femmes vassales des Dag Ghali et Aguh n Tahlé des troupeaux qui pourront assurer la survie de leurs lignées). Le récit montre également le danger de l'alliance exogame, c'est-à-dire de l'introduction d'une tente étrangère qui risque de s'accaparer des biens vitaux de l'abri où elle s'est inflitrée. Enfin il souligne la fragilité des droits d'occupation du sol qui ne sont jamais définitifs. L'histoire des confédérations touarègues qui se constituèrent et se stratifièrent suivant les vagues d'invasion successives de clans qui dominaient, intégraient ou repoussaient leurs voisins vers le sud et l'ouest, en est la démonstration.

Le territoire peut se définir comme un espace sur lequel une communauté (confédération, tribu, fraction de tribu, campement) a des droits d'usage prioritaires bien que non exclusifs. Les ressources en jeu sont essentiellement les pâturages, les points d'eau naturels ou aménagés (sources, mares, puisards, guelta, puits), le gibier, les produits de cueillette et le bois. Ces biens ne peuvent être appropriés individuellement. Leur contrôle s'établit aux différents échelons de la collectivité, représentée par un chef qui est l'amenukal ou l'ettebel au niveau de la confédération, l'amghar $n$ tawshit pour la tribu ou la fraction, l'amghar $n$ aghizven pour le campement. Chaque chef endosse la responsabilité de la gestion du territoire par rapport aux groupements voisins et aux instances hiérarchiquement supérieures.

Si les droits sur un territoire sont théoriquement définis par l'appartenance à un groupe de filiation dont l'extension peut servir idéologiquement à décrire l'ensemble social, des inégalités de fait apparaissent entre individus ou unités sociales à l'interieur de ces cercles de parenté plus ou moins fictifs.

Dans la distribution territoriale de la confédération, unité politique qui regroupe des tribus de rangs différents, la prédominance des suzerains s'affirme. Selon certaines représentations locales, c'est parce que ces derniers sont les aînés de la tente aînée, que leur revient le contrôle de l'ensemble des biens qui permettent à la grande tente de se dresser, tandis que les petites tentes sorties du grand abri ne sont directement responsables que du bien qui les fait vivre.

Ainsi, les nobles, bien que disposant eux-mêmes d'un territoire d'élection qui leur est réservé, peuvent utiliser l'ensemble du pays de la confédération, alors que les vassaux (imghad) ou autres tribus dépendantes comme celles des «affranchis * (ighawellen) sont cantonnés aux vallées qui leur ont été concédées. Ceci ne signifie pas que les vassaux n'ont pas le droit de chasser, de faire pâturer ou d'abreuver leurs troupeaux hors des limites de leur territoire si cela s'avérait utile, mais dans ce cas, ils doivent demander la permission de s'installer au responsable des lieux et quelquefois payer pour l'obtenir. De même, les tribus étrangères à la confédération devront obligatoirement acquitter des droits d'usage sur la portion de territoire qu'on leur concède, à court ou à long terme. Par exemple, la fraction Kel Tazulet des Isseqqamaren de l'Ahaggar payaient aux Kel Arikin de l'Ajjer un droit de pâture pour les oueds Assilal, Tiredjert et Tilemsi, ainsi que le droit d'abreuvoir à la source de Djuwaf dans l'Immidir (G. Gardel, 1961 : 134).

Par contre, les voyageurs, lorsqu'ils ne font que passer, ont le droit d'utiliser, 
sans permission spéciale, les pâturages d'une région ainsi que les points d'eau, une fois que les usagers habituels sont partis, c'est-à-dire après la tombée du jour (surtout quand l'eau est rare). S'ils demeurent plus de trois ou quatre jours dans une vallée, ils doivent demander l'autorisation au responsable du territoire. L'hospitalité temporaire n'est jamais refusée à un campement ou une tribu en exil. Cependant, des vallées ne leur sont allouées sur le territoire de la confédération que si leur installation ne menace pas la survie des habitants du lieu. Certains groupements se sont ainsi intégrés à une nouvelle confédération tout en conservant d'ailleurs une appellation qui marque leur origine étrangère. Si une nouvelle confédération les adopte et si elle les prend sous sa protection (en contrepartie, ils paieront alors un tribut, la tawsé), la même réception rituelle que pour la mariée qui rejoint le campement de son époux (azalay) leur est réservée par les tentes d'égale condition : un animal est sacrifié auquel on coupe les jarrets ${ }^{1}$.

Beaucoup d'unités sociales, à tous les niveaux de scission de l'ensemble confédéral (tribu, fraction, campement), portent un nom de région, de vallée ou de puits (Kel Ahaggar, Kel Ajjer; Kel Tazulet; Kel I-n-Tunin...). Il arrive souvent, à cause des migrations fréquentes des groupes, que le nom ne corresponde plus au lieu de résidence actuel (par exemple, les Kel Ferwan ou les Kel Ahaggar de l'Ayr).

Les prérogatives plus ou moins larges sur les ressources naturelles varient en fonction de leur abondance ou de leur rareté. D'une manière générale, chaque tribu réserve sur son territoire les ânes en liberté (que l'on capture pour le bât), les mouflons et les graines de cueillette : par exemple, dans l'Ahaggar, après les pluies, les différents chefs de tribu envoyaient un message au chef de confédération (amenukal) pour le prévenir qu'il était temps de mettre les vallées en défens. Si des animaux étaient surpris dans ces lieux interdits, leur propriêtaire devait payer une amende à l'amenukal. De même, pour préserver les pâturages, une mise en défens pouvait être instituée. Par exemple, une convention inter-confédérale interdisait, au moment de la repousse de l'herbe l'utilisation des pâturages salés de l'Azawagh de l'Est, fréquentés à la fois par les Kel Ayr, Kel Gress, Kel Denneg, Kel Ahaggar et quelques Kel Adghagh venus dans le Tamesna. Les mêmes restrictions s'appliquaient aux pâturages rares et fragiles des franges désertiques dont l'exploitation n'était pratiquée que lorsque l'ensemble de la collectivité était avertie de l'apparition des herbes nouvelles.

Sur les ressources territoriales placées sous sa responsabilité, chaque tente avait donc une priorité instituée par rapport aux autres tentes d'égale importance. Par contre, les tentes * aînées *, c'est-à-dire les suzerains et, parmi eux, les suzerains qui avaient droit à la chefferie et se considéraient comme les ayants droit du territoire de la confédération, pouvaient exploiter sans contrepartie ces petits territoires. Le bon usage des biens fonciers confiés à une petite tente de la confédération, comme pour les biens d'ébawél, était contrôlé par la tente aînée, qui prenait des sanctions contre les contrevenants. Enfin, l'égalité de tous les membres de la collectivité dans l'utilisation des territoires exploitables uniquement au moment des pluies et situés aux franges des territoires de confédérations, était valorisée et régie par des accords inter-confédéraux.

Sur chaque activité de production (élevage, cueillette, chasse, caravane), les dépendants (imghad, ighawellen, groupes étrangers assimilês à la confédération) devaient donner une part aux suzerains, soit immédiatement, soit annuellement sous forme de tribut (tawsê). Par exemple, les ighawellen ( affranchis *) des Kel Ghabsa, Igha- 


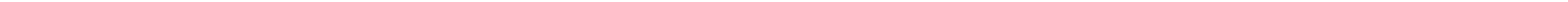


lemen et Ikarramoyen, originaires de l'Ahaggar et installés depuis longtemps dans l'Ayr, donnaient au chef des Ikazkazen, en échange de leurs droits de chasse, une certaine quantité de viande séchée de mouflon ou de gazelle ainsi que des peaux tannées. Ils apportaient également, après la cueillette, des fruits et des feuilles de palmier doum (dont on fait des vanneries).

De même, chacun sur le territoire qu'il contrôlait pouvait, à condition de ne pas entraver les activités pastorales, mettre en culture des terres (en les faisant travailler en général par des anciens esclaves ou, pour l'Ahaggar, par des agriculteurs venus du Touat), tandis que les nobles avaient le droit de cultiver où ils voulaient sur toute l'étendue du territoire de la confédération (Textes touaregs en prose, $\mathrm{n}^{\circ}$ 98). Une partie de la récolte revenait au possesseur de la terre et s'il s'agissait d'un tributaire, il devait prévoir également une autre part pour le chef des suzerains.

Les produits de la chasse étaient soumis aux mêmes règles de redistribution que les biens qui "nourrissent " la lignée. Chaque petite tente avait un droit prioritaire sur son aire de résidence par rapport aux tentes de même importance, mais la grande tente conservait la possibilité théorique de ramener le droit ou le bien dans son camp, notamment si l'usage qui en était fait ne lui convenait pas (exploitation de la zone de chasse pour un usage autre que personnel, anéantissement du gibier).

Dans l'Ahaggar, certains individus s'étaient attitrés en propre des cavernes où les mouflons viennent gîter au printemps. Si un chasseur d'un campement voisin abattait un mouflon dans l'une de ces grottes, la bête ne lui appartenait pas : il n'avait "droit qu'à la tête, la panse et les boyaux". Par contre, si le maître de la grotte (mess-is $n$ ekahem) lui en donnait la permission, le chasseur tuait la bête, et pouvait en garder la moitié pour lui (Textes touaregs en prose, $\mathrm{n}^{\circ} 110$ ). Il était bien vu que les membres des petites tentes (vassaux imghad ou assimilés) réservent de temps en temps aux suzerains dont ils dépendaient la moitié de la viande et quelque fois la peau tannée de l'animal.

Dans l'Atakor, la puissante tribu des Dag Ghali aurait interdit complètement la chasse aux autres groupes de la confédération (sauf, bien entendu, aux suzerains), cas exceptionnel, semble-t-il, de privatisation des droits sur un territoire.

Toute atteinte à l'intégrité des ressources de chaque espace alloué à une tente, était jugée et sanctionnée par le représentant de la confédération, l'amenukal ou l'ettebel. Il s'agissait notamment de la violation des vallées mises en défens (en Ahaggar, au début du siècle, selon les bêtes trouvées en train de paître, une chamelle ou cinq ou six chèvres étaient sacrifiées, Texte touaregs en prose, $\mathrm{n}^{\circ} 101$ ). De même, l'incendie volontaire était puni (cinquante coups de cravache et une amende de 250 à 500 francs environ, dans les années 1950 au Tamesna, Nicolas, $1950: 232$ ), ainsi que les coupes exagérées de bois vif (quatre chèvres d'amende par branche de gommier ou un mouton au moment de la verdure printannière en Ajjer au début du siècle, Gardel, 1961 : 131; jusqu'à dix chèvres pour une branche d'arbre à fruits comme le jujubier ou le palmier doum dans l'Ayr), ou encore le vagabondage des troupeaux dans les pâturages d'autrui et dans les cultures. Tout animal étranger trouvé sur le territoire d'une tribu devait être remis à l'amenukal ou l'ettebel, qui décidait souvent de le mettre dans les biens de la confédération (éheré $n$ ettebel) si le propriétaire demeurait inconnu ou appartenait à une tribu hostile.

Les différences $d$ 'utilisation du territoire, plus restrictives pour les vassaux que 
pour les suzerains, ont été également rapprochées des divers types d'élevage auxquels chacun se livrait : le gros bétail était réservé aux nobles, tandis que les tributaires possédaient seulement du petit bétail. En fait, ces derniers étaient autrefois chargês de garder les troupeaux de leurs maîtres qui venaient récupérer leurs bêtes (ou simplement prélever une part sur le troupeau des dépendants) au fur et à mesure de leurs besoins. La grande activité des nobles était davantage de contrôler les routes commerciales qui traversaient le pays et de percevoir la taxe de passage et de protection sur les caravanes étrangères ou encore la taxe sur les caravanes organisées par les dépendants. Leur rôle était également de protéger le territoire en prévenant les rezzous de groupes étrangers ${ }^{2}$ ou en organisant la riposte à laquelle participaient les vassaux guerriers (imghad). A cette fin, ils sillonnaient largement tout le pays sous leur contrôle tandis que les vassaux, absorbés dans les tâches d'élevage, étaient beaucoup moins mobiles.

Pour renforcer leur confédération et leur organisation défensive, les suzerains pouvaient contraindre les tribus vaincues et devenues tributaires à s'installer à un emplacement déterminé. Ainsi, les Kel Ahaggar firent venir dans le sud-ouest de leur pays les Aguh n Tahlé, originaires d'In-Gal. A une époque relativement récente, ils forcèrent les Isaqqamâren à quitter le plateau de la Tademayt qu'ils habitaient (Ch. de Foucauld, Dict., II : 536) pour rejoindre les vallées situées sur la bordure est de l'Ahaggar.

En fait, la distribution des territoires de tribus sur l'espace confédéral n'est pas laissée au hasard. Bâtis comme une tente, la société ou son territoire ne peuvent disposer n'importe où leur mât central : pour que l'édifice soit stable et solide, les dirigeants doivent toujours avoir leur place au centre, tandis que les dépendants, dont la fonction est assimilée à celle des piquets latéraux de la tente, les entourent. Dans l'Ayr, par exemple, la charpente même de la tente et les divers espaces qui la composent (par exemple tédélé, imuza, igem) se retrouvent projetés dans l'appellation des tribus et des territoires : Kel Tédélé, Kel Imezu, Igamen... (extrait de travaux inédits de Hawad sur la cosmogonie touarègue, à paraître).

\section{LE CYCLE DE MIGRATION PASTORALE}

Pour décrire son territoire, un nomade dessinera sur le sable les vallées et les puits qui s'y inscrivent, se limitant souvent à l'aire de nomadisation de sa tribu' s'il s'agit d'un amghid (tributaire) ou s'étendant à l'espace dominé par la confédération s'il s'agit d'un suzerain. Tous jeunes, les enfants apprennent à réciter par cœur le nom des vallées de leur pays ainsi que le nom des puits et de ceux qui les ont creusés. On retrouve dans ces points stratégiques définis à l'intérieur du territoire et sur lesquels d'ailleurs portent la plupart des litiges, le *chemin de l'eau " autour duquel vont s'ordonner la vie et l'espace nomades.

Le puits représente en effet la stabilité. Un proverbe dit : "Même si les vallées regorgent d'eau, la stabilité c'est le puits * (Kud ad aghlablaben ighazren asagawar $a n u)$. Le groupe de résidence, unité variable et changeante constituée souvent par des tentes apparentées, forme généralement une ou plusieurs unités de gestion du troupeau, attachées à un puits en fonction duquel vont s'organiser les déplacements tout le long de la saison sèche, tandis que pendant les brefs deux ou trois 
mois de la saison humide la forme des regroupements résidentiels sera beaucoup plus variée et souple grâce à la multiplicité des points d'eau (mares, puisards, agelmam) et des facilités relatives pour abreuver et faire pâturer les troupeaux.

Le creusage d'un puits important ne se décide, en particulier dans les régions sahariennes, qu'en accord avec l'ettebel, chef de confédération ou de grande tribu. Lorsque par exemple l'encombrement au puits devient trop important et que le partage des tours d'eau est ralenti, un nouveau puits peut être implanté à proximité de l'ancien, sur le même site. Il n'est pas rare de trouver dans un lieu donné quatre ou cinq puits côte à côte, permettant aux éleveurs d'abreuver simultanément leurs troupeaux. Par contre, l'implantation d'un grand puits dans une zone encore vierge doit répondre à certaines exigences : il est installé de préférence sur une ligne-frontière séparant les territoires de plusieurs fractions de tribu ou groupes qui ont une affinité politique. De cette manière, il servira à une collectivité étendue. Les travaux sont exécutés collectivement ou entrepris par un chef riche, capable de financer l'opération. Les "pauvres " de son entourage ou ses dépendants pourront ainsi utiliser également le puits.

D'après F. Nicolas $(1950: 233)$ * quand le puits entrepris a atteint hauteur d'homme, si l'on s'en va, le puits reste cependant à celui qui l'a implanté. Dans le cas du puits de l'année précédente, repris et recontruit, arrangé par un autre, ce puits reste au premier fondateur - sept ans de propriété temporaire - et ensuite il revient à celui qui l'a foré définitivement ». Plutôt que de "propriété », il faudrait parler ici de «droit d'usage prioritaire " et surtout de droit de gestion.

Ainsi, le nombre des puits était autrefois très contrôlé par les responsables du territoire qui pouvaient décider d'en combler un, temporairement ou définitivement, si cela s'avérait nécessaire. Une certaine distance devait être maintenue entre les stations permanentes d'eau. Seuls des puisards temporaires pouvaient être creusés de façon plus anarchique dans les vallées bien alimentées en eau. De même, les jardins ne pouvaient pas être implantés n'importe où et encore moins au cœur des pâturages comme cela se pratique aujourd'hui.

Cette économie d'aménagement des points d'eau n'avait rien à voir avec un manque de moyens (soulignons d'ailleurs la perfection des coffrages en pierre de l'Adghagh, par exemple, dont la robustesse dépasse de beaucoup celle des maçonneries modernes qui utilisent des matériaux importés et coûteux). En effet, les éleveurs savent bien qu'une multiplication et un éparpillement à courte distance des points d'eau pérennes ont un effet très dangereux sur la gestion des ressources en empêchant la constitution des pâturages de réserve ${ }^{3}$. D'autre part, à l'époque des rezzous, cela aurait facilité aux pillards l'accès du pays.

Le puits représente donc un véritable "centre" de rencontre, un point nodal entre plusieurs fractions ou tribus qui l'utilisent collectivement. Il est creusé au croisement de leurs territoires. Chacun de ces groupes dispose, pour extraire l'eau, d'une portion de margelle (tafult) ou encore d'une fourche qui soutient la poulie, orientée du côté de son «pays". La répartition et l'attribution des espaces et des fourches autour de la margelle du puits livrent ainsi parfaitement la distribution des territoires rayonnant autour de ce point de référence inamovible.

Dès la fin de l'année écologique, c'est-à-dire à l'automne (gharat), quand apparaît l'étoile Ghadet et que souffle le vent agzer qui sèche les outres de lait et donc les pâturages, les éleveurs se replient vers leur territoire permanent et s'établissent 
près du puits. Chacun réintègre sa vallée d'élection et retrouve les affaires qu'il y avait laissées pour ne pas alourdir ses bagages. Ce retour (éras) a ramené les tentes à leur point de départ et marque le commencement d'un nouveau cycle de migration pastorale.

A cette époque, les nomades sont capables d'évaluer la qualité des pâturages dont ils disposeront pour l'année à venir. Ils vont alors structurer leurs activités suivant la situation. L'exploitation du territoire, la séparation des troupeaux (une partie réservée aux voyages caravaniers, une destinée à la vente, une autre conduite dans les pâturages lointains, une dernière restant à proximité du campement), ainsi que l'approvisionnement et les mouvements caravaniers sont alors organisés et décidés à l'échelle de chaque tribu.

Les mouvements des campements à l'intérieur du territoire suivront le rythme des saisons et l'état de la flore classée en plusieurs catégories de pâturage (appelé en tayrt tamedint; le pluriel de ce terme, timdan ou cimdan, s'emploie pour désigner des pâturages peu abondants). Du puits à la tente, de la tente aux pâturages, ces va-et-vient, journaliers, hebdomadaires ou annuels forment chaque fois un cycle, dont les étapes saisonnières concrètes seront données ici en prenant l'exemple de l'Ayr occidental ${ }^{4}$.

Au début de l'automne, donc, après la saison des pluies, les tentes s'installent au cœur des teshawin ("les écorces") dont la tige est creuse et qui poussent sur des terrains inondés par les crues : 1'herbe est alors sèche et se dénomme, dans cet état, aghar.

Ces pâturages sont consommés en priorité car ils ne durent pas, menacés par les premiers vents qui les arrachent de terre et les emportent. Dès le début du mois de mars, ils ont disparu. Les feuilles des arbres qui sont tombées (awakala) et servent à nourrir le petit bétail sont classées également parmi les teshawin et consommées en mars-avril.

$\mathrm{Au}$ fur et à mesure que ces pâturages s'épuisent, le campement se déplace et pousse le troupeau vers l'alemoz, qui apparaît, entre les plaines et les lits de vallées traversées par les crues, sur les berges hautes en terrain sableux ou peu argileux où l'eau ne stagne pas. Alemoz se compose de petites tiges fines terminées par un piquant (comme par exemple teghamut, tazmey...) et souvent regroupées en touffe. Ce pâturage peut se conserver un an ou deux. On l'appelle alors ariko. Il est consommé de préférence d'avril à juin car ses épines tombent alors, emportées par le vent. On l'économise car il résiste parfaitement aux bourrasques et au soleil.

A partir de juin ou juillet, les tentes gagnent les extrémités du territoire. Le troupeau évolue dans les tizewin («tiges $)$ ). Ce pâturage à tiges résistantes se présente sous forme de touffes (comme afazu, tullult, tébaremt, gerfis, âmasa...). C'est le fourrage de réserve dont font provision les caravaniers qui entreprennent leur voyage. Faisant partie de la même catégorie de pâturage, les imerkerjaj désignent de petits arbustes poussant sur les terrains érodés par le vent et qui restent vivaces toute l'année. Toutes ces plantes représentent un pâturage d'appoint, assez lourd, qui donne du mucilage (ésak), et qui est utilisé surtout en juin et juillet, de préférence une fois que les premières pluies l'ont arrosé et assoupli. Consommé à la fin de l'été, il est la dernière pitance et le dernier recours de la saison sèche.

Si la pluie tarde, les troupeaux restent entre les imerkerjaj et les vallées où cer- 
tains arbres (comme tadant ou abezgin) demeurent verts.

Si les grandes pluies ne sont pas arrivées à partir de septembre, les pluies à venir ne pourront faire pousser que les teshawin, tandis que la croissance de alemoz sera compromise. Par contre, toute pluie, même légère, peut faire verdir les tizewin et les imerkerjaj car les racines sont déjà constituées et le buisson existe même s'il est sec.

Les différentes étapes menant d'une catégorie de pâturage à une autre ont progressivement éloigné le campement du puits. Dès les premières pluies, à partir d'août, les tentes s'installent sur les berges des oueds, en bordure des teshawin : cette herbe est alors fraîche et constitue un pâturage fragile, facile à casser, dont les tiges molles s'étalent sur le sol au lieu de pousser dru. On l'appelle akasa.

Alors débute la saison faste (ghafet), celle de l'abondance de l'eau et des pâturages. Les tribus se retrouvent aux frontières de leurs territoires et se réunissent pour se rendre ensemble vers d'autres pâturages d'akasa, loin de leurs vallées de résidence. Au contraire de la saison sèche où les déplacements se font sur un rayon très court, les parcours s'allongent et certains groupes font des centaines de kilomètres pour atteindre ces nouveaux pâturages. Hormis les personnes trop âgées pour se déplacer ainsi que les esclaves qui restent pour la cueillette des graines sauvages ou les gens très pauvres qui n'ont que quelques têtes de petit bétail, tous les campements doivent quitter les vallées pour se rendre dans les plaines. Ils atteignent ces zones fréquentées uniquement pendant les pluies et où personne autrefois n'habitait en permanence. Dans ces aires gérées par des accords inter-confédéraux (comme par exemple les pâturages de la cure salée des grandes plaines d'Azawagh), aucun groupe n'est prioritaire. C'est à cette époque de l'année que le cycle pastoral s'achève, apothéose couronnée par les grandes fêtes qui rassemblent la communauté élargie et renouent les liens de solidarité avant la prochaine dispersion. La fin du parcours qui précède la naissance d'un nouveau cycle est le moment par excellence de l'équilibre et de l'harmonie, où les points de référence classiques, les étapes ordonnées et obligatoires de la migration pastorale, sont soudain confondus. Eau, tente et pâturages se trouvent regroupés dans le même lieu; les troupeaux de petit ou gros bétail sont mêlés; les différentes tribus et confédérations s'enchevêtrent; les lois strictes des transhumances de la saison sèche, devenues inutiles, s'évanouissent et cèdent la place à des mouvements plus débridés. Mais dès que l'herbe commence à sécher, annonçant la fin de l'hivernage, les campements se replient avec une certaine précipitation et regagnent leurs vallées.

Au contraire de la saison humide où l'eau et les pâturages, partout présents et abondants, peuvent être utilisés sans organisation sévère, pendant la saison sèche, diverses aires de pacage sont établies et distinguées suivant leur exploitation par les unités de gardiennage des troupeaux. A chaque nouvelle avancée du campement, ces espaces successifs qui permettent d'économiser les pâturages, se rééchelonnent à partir des tentes. L'étagement de ces zones dont l'épicentre est le campement sert aussi à désigner les étapes qu'accomplit le voyageur qui entame son parcours à l'aube.

Ainsi, ce dernier va d'abord traverser les premiers pâturages où paissent, sous la garde de jeunes bergers, chèvres, moutons, vaches (dans le sud du pays), ânes et chameaux entravés, chamelons qui rentrent chaque soir au campement. 


\section{0 / H. Claudot-Hawad}

Asember est l'espace le plus proche, le lieu où les animaux pâturent la nuit autour du campement.

Amasadon représente les pâturages environnants qui restent visibles du campement et que fréquente le petit bétail pendant la journée.

Plus éloigné des tentes, eshezvul marque les pâturages atteints par le petit bétail parti le matin à l'aube et arrivé à l'heure de la sieste, c'est-à-dire à une étape de marche appelée takelawt.

C'est également à l'heure de la sieste que le voyageur arrivera dans les pâturages de réserve fréquentés en saison sèche uniquement par les troupeaux transhumants et les animaux qui peuvent s'éloigner quelques jours des points d'eau.

Ici commence l'aghaytum divisé lui-même en plusieurs sous-ensembles : le premier est l'esenteg, lieu que le troupeau de chamelles parti au petit matin avant la naissance de l'aube atteint à midi, tandis que le deuxième, appelé tiseseklaw, n'est atteint par le gros bétail que vers une heure de l'après-midi (étape takelawt). Les animaux qui rentrent le soir au campement n'arrivent qu'au début de cette aire de tiseseklaw.

Enfin, le voyageur, à deux jours de marche environ du campement, reconnaîtra les pâturages lointains de l'aghaytum proprement dit, que seuls les chameaux laissés en liberté ou égarés fréquentent, car ils peuvent résister longtemps à la soif. Cet espace est également une zone de chasse et de cueillette de graines sauvages. Les nomades y cachent leurs réserves alimentaires dans des greniers ou des abris sous roche.

Souvent un rocher volumineux posé sur trois pierres plus petites (tasakadawt) borne l'espace d'une tribu et, sur sa paroi, les marques de bétail de la communauté sont gravées. Pour s'amuser, les jeunes gens d'un groupe voisin peuvent essayer de déplacer le roc, toujours très lourd, conquérant ainsi, symboliquement, une portion de sol à leur avantage.

Pour le voyageur, la zone nommée ameshedwi est la bordure de l'aghaytum. Loin du puits, loin de la vie, cette aire marque les frontières du territoire qu'il vient de parcourir, la limite de l'espace domestiqué et familier, le début de l'inconnu.

Si le puits est le point nodal où les territoires se rencontrent, où chacun inserre un angle qui prend place dans le cercle et à partir duquel s'organise l'espace nomade, l'aghaytum représente l'autre extrémité du territoire qui s'ouvre sur l'aghaytum d'un autre espace de tribu ou encore, s'il s'agit d'un territoire de "bordure * ou d'angle, s'achève sur le «désert ". Au moment de l'hivernage seulement, l'aghaytum se transforme à son tour en lieu de rencontre et l'essuf, chassé du désert, s'installe de l'autre côté du territoire, sur les puits et les vallées délaissées. Par contre, en saison sèche, les tentes reviennent autour des puits et l'aghaytum ne dépasse plus les lieux de campement de la période des pluies.

Les mouvances des tentes qui s'écartent du puits pour s'approcher des frontières du territoire, sont également une manière de repousser à chaque étape nouvelle l'kextérieur " dans des confins de plus en plus lointains. Mais en même temps, pour que cette solitude ne s'installe pas dans leur sillage, dans les lieux délaissés, il convient de ne jamais interrompre le mouvement nomade. La migration est ainsi conquête perpétuelle contre l'envahissement de l'essuf qui croise chaque point du parcours et qui, à la fois, précède et suit les voyageurs, se glissant dans leurs traces 
et occupant tous les bivouacs abandonnés. Cependant, l'essuf fait partie intégrante de l'univers et lorsque le campement se déplace, c'est aussi pour lui laisser sa "part " ou son «tour ».

Semblables au parcours qui mène le nomade et son troupeau de la tente au puits et du puits à la tente, les migrations pastorales annuelles ou encore la vie et l'audelà de la vie sont vus comme un enchaînement de cycles qui conduisent à ce moment privilégié de transition et d'harmonie, à cet instant d'équilibre qui succède à la fin d'une action et devance le début d'une autre. Comme des pèlerins s'acheminent sur "la route qui mène au puits ", les éléments de l'univers traversent ainsi, infatigables, vallées et plateaux arides jusqu'à atteindre l'étape ultime du repos, point de départ d'un nouveau voyage. S'immobiliser, se fixer, se sédentariser, serait interrompre la marche de l'univers, échapper à l'ordre cosmique, se désintégrer dans un horizon inconnu.

\section{NOTES}

1. Ce rituel d'accueil, probablement très ancien et aujourd'hui freiné par la réprobation des marabouts islamiques, est pratiqué dans de nombreux groupes touaregs (Ajjer, Ayr, Issaqqamaren de l'Ahaggar...). Il a été également observé dans d'autres sociétés berbères comme par exemple les Iqar'iyen du Maroc (R. Jamous, Honneur et Baraka - Les structures politiques et religieuses des Iqar'iyen à la fin du XIX' siècle, thèse de $3^{e}$ cycle, EHESS, 1978, 297 p. ronéo) ou encore chez les Maures (G. Feral, Le tambour des sables, éd. France-Empire, 1983 : photo hors texte de la «targuiba*). Il est également connu dans le monde pré-islamique et appelé aqara, verbe devenu synonyme de * sacrifier par générosité * (J.-J. Schmidt, Les Mou'allaqat, Seghers, 1978 : 69 , note 16$)$.

2. Sur ce point, voir $H$. Claudot et $M$. Hawad, 1982.

3. Depuis que les nomades ont été privés de leurs droits de gestion du sol au profit des interventions plus ou moins expertes d'organismes internationaux ou locaux, cette peur d'un déséquilibre entre eau et pâturage, envisagé sur la durée du cycle de transhumance, semble avoir complètement disparue. La prolification des forages à l'ouest de l'Ayr, par exemple, a abouti à une véritable destruction de la zone, en provoquant une telle concentration des hommes et des troupeaux que la conservation de pâturages de réserve est devenue impossible. Ajoutons que beaucoup de parcours nomades ont été raccourcis et amputés dès la colonisation française pour isoler certaines tribus et renforcer la surveillance et le contrôle des zones nomades, mesures qui ont pesé gravement sur la gestion des pâturages et l'étagement des aires d'exploitation pastorale.

4. Il existe différents travaux sur le déploiement géographique et écologique des migrations pastorales de certains groupes. Voir par exemple E. Bernus, 1974 et 1981, A. Bourgeot, 1978.

\section{NOTATION PHONÉTIQUE}

La notation phonétique adoptée se réfere en grande partie à l'alphabet phonétique international. Les variantes en sont les suivantes :

gh pour la fricative vélaire sonore

kh pour la fricative vélaire sourde

i pour la fricative palato-alvéolaire sonore

sh pour la fricative palato-alvéolaire sourde

y pour la semi-voyelle pré-palatale sonore

é pour la voyelle antérieure non-arrondie mi-fermée

e pour la voyelle neutre

Les termes touaregs cités dans cet article sont en tayrt (dialecte de l'Ayr). 


\section{2 / H. Claudot-Hawad}

\section{BIBLIOGRAPHIE}

BERNUS (E.), 1974, * Les Illabakan (Niger), une tribu touarègue sahélienne et son aire de nomadisation *, Atlas des structures agraires au sud du Sahara, n 10, Paris, ORSTOM/Mouton, 116 p., 14 cartes h.t.

Bernus (E.), 1981, Touaregs nigériens, Unité culturelle et diversité régionale d'un peuple pasteur, Paris, ORSTOM, 507 p., 5 cartes h.t.

Bourgeot (A.), 1978, Étude de l'évolution d'un système d'exploitation sahélien du Mali, Rapport de mission, A.C.C. - Lutte contre l'aridité en milieu tropical, 44 p.

Chaker (S.), Claudot (H.), Gast (M.) éd., 1985, Textes touaregs en prose de Ch. de Foucauld et A. de Calassanti-Motylinski, Édisud, Aix-en-Provence, 359 p.

Claudor (H.), 1980, * Les terminologies de parenté, leurs variations, leur interprétation *, Colloque de Gif sur la parenté touarègue, 23-26 sept. 1980, travaux du LAPMO.

Claudot (H.), 1982, La sémantique au service de l'anthropologie, Recherche méthodologique et application à l'étude de la parenté chez les Touaregs de l'Ahaggar, éd. du CNRS, Paris, 273 p.

Claudor (H.), 1983, * Le discours légaliste de la parenté *, Annuaire de l'Afrique du Nord, 533-543.

Claudot (H.), 1986, *A qui sert l'unifiliation?", in Bernus (S.), Bonte (P.), Brock (L.) et Claudot (H.) éds., Le fils et le neveu, feux et enjeux de la parenté touarègue, Cambridge University Press/MSH, 191-205.

Claudot (H.) et Hawad (M.), 1982, « Coups et contre-coups : l'honneur en jeu chez les Touaregs *, Annuaire de l'Afrique du Nord, n 21, Paris, CNRS, 793-808.

Claudot (H.) et Hawad (M.), 1984a, "Ébawél/essuf, les notions d"intérieur" et d"“extérieur" dans la société touarègue *, R.O.M.M., $\mathrm{n}^{\circ} 38,171-180$.

Claudot (H.) et Hawad (M.), 1984b, *Le lait nourricier de la société ou la prolongation de soi chez les Touaregs ", in Gast (M.) éd., Hériter en pays islamique, CNRS, à paraître, Aix-en-Provence, 1987.

Duveyrier (H.), 1864, Les Touaregs du Nord, Paris, Challamel Aîné, 488 p.

Foucauld (Père Ch. de), 1951-52, Dictionnaire touareg-français, Dialecte de l'Ahaggar, Imprimerie Nationale, Paris, 4 vol.

Gardel (G.), 1961, Les Touaregs Ajjer, éd. Baconnier, Alger, 388 p.

Nicolas (F.), 1950, Tamesna, Les Ioullemmeden de l'est ou Touâreg Kel Dinnik, Imprimerie Nationale, Paris, 279 p.

Production pastorale et société, bulletin de l'équipe écologie et anthropologie des sociétés pastorales, MSH, Paris : Debat sur l'organisation territoriale et la notion de territoire dans les sociétés de pasteurs nomades, $n^{\circ} 6,8,9,10,11$ en particulier. 